\title{
Reflection of plane waves in an initially stressed perfectly conducting transversely isotropic solid half-space
}

\author{
Baljeet Singh ${ }^{1}$ and Anand Kumar Yadav ${ }^{2}$ \\ ${ }^{1}$ Department of Mathematics, Post Graduate Government College, Sector 11, Chandigarh, India. \\ ${ }^{2}$ Department of Mathematics, Shishu Niketan Model Senior Secondary School, Chandigarh, India. \\ *Corresponding author. e-mail: dr_baljeet@hotmail.com
}

Reflection of plane waves is studied at a free surface of a perfectly conducting transversely isotropic elastic solid half-space with initial stress. The governing equations are solved to obtain the velocity equation which indicates the existence of two quasi planar waves in the medium. Reflection coefficients and energy ratios for reflected $\mathrm{qP}$ and qSV waves are derived and computed numerically for a particular material. Effects of the initial stress and magnetic field are shown graphically on these reflection coefficients and energy ratios.

\section{Notations}

$\begin{array}{ll}u_{i} & \text { Components of displacement vector } \\ \rho & \text { Density of the medium } \\ \vec{J} & \text { Electric current density vector } \\ \vec{B} & \text { Magnetic induction vector } \\ \vec{H} & \text { Total magnetic field vector } \\ \vec{E} & \text { Electric field vector } \\ \sigma & \text { Electric conductivity of the medium } \\ \omega & \text { Circular frequency } \\ \mu_{e} & \text { Magnetic permeability of the medium } \\ \theta & \text { Angle of propagation measured from } \\ & \text { normal to the half-space } \\ \delta_{i j} & \text { Kronecker delta } \\ \vec{F} & \text { Body force vector } \\ p_{0} & \text { Hydrostatic state of stress, where } \\ & p_{0}<0 \text { stands for tension and } p_{0}>0 \\ e_{i j} & \text { stands for compression } \\ \tau_{i j} & \text { Strain components } \\ A, F, L, C, N & \text { Stress components } \\ \text { Superscript } 0 & \text { Initial equilibrium state } \\ \sim & \text { Incremental terms }\end{array}$

\section{Introduction}

In the last 2-3 decades, the concept of electromagnetic composite materials has arisen. Such composites can exhibit field coupling that is not present in any of the monolithic constituent materials. These so called 'Smart' materials, and composites have applications in ultrasonic imaging devices, sensors, actuators, transducers and many other emerging components. Magneto-electro-elastic materials are used in various applications. Due to the ability of converting energy from one kind to another (among mechanical, electric and magnetic energies), these materials have been used in high-tech areas such as lasers, supersonic devices, microwave and infrared applications. Furthermore, magnetoelectro-elastic materials exhibit coupling behaviour among mechanical, electric, and magnetic fields and are inherently anisotropic.

The term 'initial stress' is meant by stresses developed in a medium before it is being used for study. The earth is an initially stressed medium, due to presence of external loading, slow process

Keywords. Anisotropy; magneto-elasticity; initial stress; plane waves; reflection; reflection coefficient and energy ratio. 
of creep and gravitational field, due to variation of temperature, weight, overburden layer and hydrostatic tension or compression and largeness, process of quenching, shot peening and cold working, differential external forces, etc. Therefore, considerable amount of stresses (initial stresses) remain naturally present in the layers. These stresses may have significant influence on elastic waves produced by earthquake or explosions and also in the stability of the medium. It is therefore of much interest to study the influence of these stresses on the propagation of stress waves as initially stressed materials, which have various applications. An initially stressed and magneto-elastic nature of earth affects the propagation of seismic waves.

The problems on reflection and refraction phenomena in media with initial stresses, anisotropy, thermal, electric and magnetic fields are studied by many researchers, for example, Knott (1899); Gutenberg (1944); Tooly et al. (1965); Yu and Tang (1966); Acharya (1970); De and Sengupta (1972); Auld (1973); Achenbach (1976); Keith and Crampin (1977); Dey and Addy (1979); Tolstoy (1982); Norris (1983); Pal and Chattopadhyay (1984); Das et al. (1994); Rakshit and Sengupta (1998); Ahmad and Khan (2001); Chattopadhyay and Rogerson (2001); Selim and Ahmed (2006); Sharma (2007); Singh (2010) and many more.

Wave reflection and transmission at interfaces between two similar or dissimilar materials is of interest in many fields, e.g., composite engineering, geology, seismology, seismic exploration, control systems and acoustics. But, comparatively little investigation has been made in an anisotropic media with initial stresses and magnetic field. The present paper investigates the effects of the initial stress and magnetic field on the reflection coefficients and energy ratios of reflected waves in a perfectly conducting initially stressed transversely isotropic elastic solid half-space. The present work is supposed to be useful in further studies of wave propagation in the more realistic models which have been extensively used in many engineering and industrial applications such as computer technology, actuators, sensors, radio, intelligent structures, ultrasonic, etc. This investigation is also relevant to acoustic device application of piezoelectric composite structures.

\section{Basic equations}

Consider a homogenous perfectly electrically conducting charge free transversely isotropic medium subjected to an initially hydrostatic state of stress (compression) and permeated by a constant (time and position independent) magnetic field $\vec{H}=$ $\left(0, H_{0}, 0\right)$. Consider the plane wave propagating in $x_{1} x_{3}$ plane, $x_{3}$ is vertical axis pointing downwards and displacement components are $\vec{u}=\left(u_{1}, 0, u_{3}\right)$ and $\partial / \partial x_{2}=0$.

The Maxwell's electromagnetic equations are:

$$
\begin{gathered}
\nabla \times \vec{H}=\vec{J}, \quad \nabla \times \vec{E}=-\frac{\partial \vec{B}}{\partial t}, \\
\nabla \cdot \vec{B}=0, \quad \vec{B}=\mu_{e} \vec{H}_{0} .
\end{gathered}
$$

The generalized Ohm's laws in deformable continuum is:

$$
\vec{J}=\sigma\left[\vec{E}+\frac{\partial \vec{u}}{\partial t} \times \vec{B}\right]
$$

where $\vec{E}$ is the electric intensity vector, $\vec{H}$ is the constant magnetic field $\vec{H}\left(0, H_{0}, 0\right), \vec{B}$ is the magnetic induction vector, $\vec{J}$ is the current density vector, $\mu_{e}$ represents the magnetic permeability, $\sigma$ is the electric conductivity, $\vec{h}\left(h_{1}, h_{2}, h_{3}\right)$ is change in the basic magnetic field, $\vec{u}$ is the displacement vector, and $t$ denotes time.

From equations (1) and (2), it follows that:

$$
\frac{1}{\sigma \mu_{e}} \nabla^{2} \vec{H}=\left[\frac{\partial \vec{H}}{\partial t}-\nabla \times\left(\frac{\partial \vec{u}}{\partial t} \times \vec{H}\right)\right]
$$

where

$$
\begin{aligned}
\nabla \times & \left(\frac{\partial \vec{u}}{\partial t} \times \vec{H}\right) \\
& =\left(0,-H_{0}\left\{\frac{\partial}{\partial x_{1}}\left(\frac{\partial u_{1}}{\partial t}\right)+\frac{\partial}{\partial x_{3}}\left(\frac{\partial u_{3}}{\partial t}\right)\right\}, 0\right) .
\end{aligned}
$$

In component form, equation (3) can be written as:

$$
\begin{aligned}
\frac{\partial H_{x_{1}}}{\partial t}= & \frac{1}{\sigma \mu_{e}} \nabla^{2} H_{x_{1}} \\
\frac{\partial H_{x_{2}}}{\partial t}= & \frac{1}{\sigma \mu_{e}} \nabla^{2} H_{x_{2}} \\
& -H_{0}\left\{\frac{\partial}{\partial x_{1}}\left(\frac{\partial u_{1}}{\partial t}\right)+\frac{\partial}{\partial x_{3}}\left(\frac{\partial u_{3}}{\partial t}\right)\right\} \\
\frac{\partial H_{x_{3}}}{\partial t}= & \frac{1}{\sigma \mu_{e}} \nabla^{2} H_{x_{3}} .
\end{aligned}
$$

For perfectly conducting medium $(\sigma \rightarrow \infty)$, equation (4) becomes:

$$
\begin{aligned}
& \frac{\partial H_{x_{1}}}{\partial t}=0, \quad \frac{\partial H_{x_{3}}}{\partial t}=0, \\
& \frac{\partial H_{x_{2}}}{\partial t}=-H_{0}\left\{\frac{\partial}{\partial x_{1}}\left(\frac{\partial u_{1}}{\partial t}\right)+\frac{\partial}{\partial x_{3}}\left(\frac{\partial u_{3}}{\partial t}\right)\right\} .
\end{aligned}
$$

We assume that the primary magnetic field is uniform throughout the space. It is clear from equation (5) that there is no perturbation in $H_{x_{1}}$ and $H_{x_{3}}$ but from equation (6) it is clear that there is perturbation in $H_{x_{2}}$. Therefore, taking small 
perturbations $h_{2}$ in $H_{x_{2}}$ and integrating equations (5) and (6), we get:

$$
\begin{aligned}
& H_{x_{1}}=0, \quad H_{x_{3}}=0 \\
& H_{x_{2}}=-H_{0}\left\{\frac{\partial u_{1}}{\partial x_{1}}+\frac{\partial u_{3}}{\partial x_{3}}\right\}
\end{aligned}
$$

Therefore,

$$
h_{1}=0, \quad h_{2}=-H_{0}\left\{\frac{\partial u_{1}}{\partial x_{1}}+\frac{\partial u_{3}}{\partial x_{3}}\right\}, \quad h_{3}=0 .
$$

From, relation $\vec{F}_{i}=\mu_{e}(\operatorname{Curl} \vec{h} \times \vec{H})$, we obtain:

$$
\begin{aligned}
& F_{1}=(\vec{J} \times \vec{B})_{1}=\mu_{e} H_{0}^{2}\left(\frac{\partial^{2} u_{1}}{\partial x_{1}^{2}}+\frac{\partial^{2} u_{3}}{\partial x_{1} \partial x_{3}}\right), \\
& F_{2}=(\vec{J} \times \vec{B})_{2}=0, \\
& F_{3}=(\vec{J} \times \vec{B})_{3}=\mu_{e} H_{0}^{2}\left(\frac{\partial^{2} u_{1}}{\partial x_{1} \partial x_{3}}+\frac{\partial^{2} u_{3}}{\partial x_{3}^{2}}\right) .
\end{aligned}
$$

We have considered the medium as perfectly conducting medium. If the medium is not perfectly conducting, then magnetic field applied get perturbated in directions of all the three co-ordinate axes and because of resistivity of the medium, attenuations will take place. The theory of incremental stress and strain was developed by Biot (1965). Following $\mathrm{Yu}$ and Tang (1966), the equations of motion for electrically conducting charge free elastic solid under initial stress permeated by an electromagnetic field, which are based on the finite strain theory when body is subjected to small perturbation are:

$$
\rho \frac{\partial^{2} u_{i}}{\partial t^{2}}=\frac{\partial}{\partial x_{j}}\left[\tau_{j k}\left(\delta_{j k}+\frac{\partial u_{i}}{\partial x_{j}}\right)\right]+(\vec{J} \times \vec{B})_{i}+\vec{F}_{i}
$$

where

$$
\begin{aligned}
\tau_{j k} & =\tau_{j k}^{0}\left(x_{1}, 0, x_{3}\right)+\widetilde{\tau}_{j k}\left(x_{1}, 0, x_{3}, t\right), \\
F_{i} & =F_{i}^{0}\left(x_{1}, 0, x_{3}\right)+\widetilde{F}_{i}\left(x_{1}, 0, x_{3}, t\right) \\
H_{i} & =H_{i}^{0}\left(x_{1}, 0, x_{3}\right)+\widetilde{H}_{i}\left(x_{1}, 0, x_{3}, t\right) .
\end{aligned}
$$

Following Love (1942), the components $\tau_{i j}$ of stress tensor are:

$$
\begin{aligned}
\tau_{11} & =A \frac{\partial u_{1}}{\partial x_{1}}+F \frac{\partial u_{3}}{\partial x_{3}} \\
\tau_{22} & =(A-2 N) \frac{\partial u_{1}}{\partial x_{1}}+F \frac{\partial u_{3}}{\partial x_{3}}, \\
\tau_{33} & =F \frac{\partial u_{1}}{\partial x_{1}}+C \frac{\partial u_{3}}{\partial x_{3}} \\
\tau_{13} & =L\left(\frac{\partial u_{1}}{\partial x_{3}}+\frac{\partial u_{3}}{\partial x_{1}}\right) .
\end{aligned}
$$

With the help of equations (7-9), the equations of motion for perfectly conducting transversely isotropic solid under the initial state of hydrostatic stress (compression) and in the presence of constant magnetic field $\vec{H}\left(0, H_{0}, 0\right)$ as:

$$
\begin{gathered}
A \frac{\partial^{2} u_{1}}{\partial x_{1}^{2}}+(F+L) \frac{\partial^{2} u_{3}}{\partial x_{1} \partial x_{3}}+L \frac{\partial^{2} u_{1}}{\partial x_{3}^{2}}-p_{0}\left(\frac{\partial^{2} u_{1}}{\partial x_{1}^{2}}+\frac{\partial^{2} u_{1}}{\partial x_{3}^{2}}\right) \\
+\mu_{e} H_{0}^{2}\left(\frac{\partial^{2} u_{1}}{\partial x_{1}^{2}}+\frac{\partial^{2} u_{3}}{\partial x_{1} \partial x_{3}}\right)=\rho \frac{\partial^{2} u_{1}}{\partial t^{2}}
\end{gathered}
$$

$$
\begin{gathered}
L \frac{\partial^{2} u_{3}}{\partial x_{1}^{2}}+(F+L) \frac{\partial^{2} u_{1}}{\partial x_{1} \partial x_{3}}+C \frac{\partial^{2} u_{3}}{\partial x_{3}^{2}}-p_{0}\left(\frac{\partial^{2} u_{3}}{\partial x_{1}^{2}}+\frac{\partial^{2} u_{3}}{\partial x_{3}^{2}}\right) \\
+\mu_{e} H_{0}^{2}\left(\frac{\partial^{2} u_{1}}{\partial x_{1} \partial x_{3}}+\frac{\partial^{2} u_{3}}{\partial x_{3}^{2}}\right)=\rho \frac{\partial^{2} u_{3}}{\partial t^{2}} .
\end{gathered}
$$

\section{Plane wave solution}

The appropriate displacement potentials are:

$$
\begin{aligned}
& u_{1}=A^{\prime} \exp \left\{i k\left(x_{1} \sin \theta+x_{3} \cos \theta-V t\right)\right\} \\
& u_{3}=B^{\prime} \exp \left\{i k\left(x_{1} \sin \theta+x_{3} \cos \theta-V t\right)\right\}
\end{aligned}
$$

Using equations (12) and (13) in equations (10) and (11), we obtain:

$$
\begin{aligned}
\left(P_{1}-\zeta\right) A^{\prime}+Q B^{\prime} & =0 \\
Q A^{\prime}+\left(P_{2}-\zeta\right) B^{\prime} & =0
\end{aligned}
$$

where

$$
\begin{aligned}
& P_{1}=A \sin ^{2} \theta+L \cos ^{2} \theta-p_{0}+\mu_{e} H_{0}^{2} \sin ^{2} \theta, \\
& P_{2}=L \sin ^{2} \theta+C \cos ^{2} \theta-p_{0}+\mu_{e} H_{0}^{2} \cos ^{2} \theta, \\
& Q=\left(F+L+\mu_{e} H_{0}^{2}\right) \sin \theta \cos \theta .
\end{aligned}
$$

For the existence of non-trivial solution, the determinant of the coefficients of $A^{\prime}$ and $B^{\prime}$ must vanish, i.e.,

$$
\zeta^{2}-\left(P_{1}+P_{2}\right) \zeta+\left(P_{1} P_{2}-Q^{2}\right)=0
$$

where $\zeta=\rho V^{2}$. The two roots of the equation (16) are written as:

$$
\begin{aligned}
& \zeta_{1}=\frac{\left(P_{1}+P_{2}\right)+\sqrt{\left(P_{1}-P_{2}\right)^{2}+4 Q^{2}}}{2}, \\
& \zeta_{2}=\frac{\left(P_{1}+P_{2}\right)-\sqrt{\left(P_{1}-P_{2}\right)^{2}+4 Q^{2}}}{2}
\end{aligned}
$$

which correspond to the speeds of propagation of quasi-P (qP) and quasi-SV (qSV) waves, respectively. 


\section{Reflection from a free surface}

A transversely isotropic perfectly conducting initially stressed solid half space $\left(x_{3}>0\right)$ is considered. The positive $x_{3}$ is taken into the half space. Reflection of $\mathrm{qP}$ or $\mathrm{qSV}$ wave will generate two reflected $\mathrm{qP}$ and reflected $\mathrm{qSV}$ waves in the half space. The complete geometry of the problem is shown in figure 1 . The appropriate displacement potentials are:

$$
\begin{aligned}
u_{1}= & A_{0} \exp \left\{i k_{0}\left(x_{1} \sin \theta_{0}-x_{3} \cos \theta_{0}-V_{0} t\right)\right\} \\
& +A_{1} \exp \left\{i k_{1}\left(x_{1} \sin \theta_{1}+x_{3} \cos \theta_{1}-V_{1} t\right)\right\} \\
& +A_{2} \exp \left\{i k_{2}\left(x_{1} \sin \theta_{2}+x_{3} \cos \theta_{2}-V_{2} t\right)\right\},
\end{aligned}
$$

$$
\begin{aligned}
u_{3}= & F_{0} A_{0} \exp \left\{i k_{0}\left(x_{1} \sin \theta_{0}-x_{3} \cos \theta_{0}-V_{0} t\right)\right\} \\
& +F_{1} A_{1} \exp \left\{i k_{1}\left(x_{1} \sin \theta_{1}+x_{3} \cos \theta_{1}-V_{1} t\right)\right\} \\
& +F_{2} A_{2} \exp \left\{i k_{2}\left(x_{1} \sin \theta_{2}+x_{3} \cos \theta_{2}-V_{2} t\right)\right\},
\end{aligned}
$$

where

$$
\begin{aligned}
F_{0}= & \frac{\left(F+L+\mu_{e} H_{0}^{2}\right) \sin \theta_{0} \cos \theta_{0}}{L \sin ^{2} \theta_{0}+C \cos ^{2} \theta_{0}+\mu_{e} H_{0}^{2} \cos ^{2} \theta_{0}-p_{0}-\zeta_{0}}, \\
F_{\ell}= & \frac{-\left(F+L+\mu_{e} H_{0}^{2}\right) \sin \theta_{\ell} \cos \theta_{\ell}}{L \sin ^{2} \theta_{\ell}+C \cos ^{2} \theta_{\ell}+\mu_{e} H_{0}^{2} \cos ^{2} \theta_{\ell}-p_{0}-\zeta_{\ell}}, \\
& \quad(\ell=1,2) .
\end{aligned}
$$

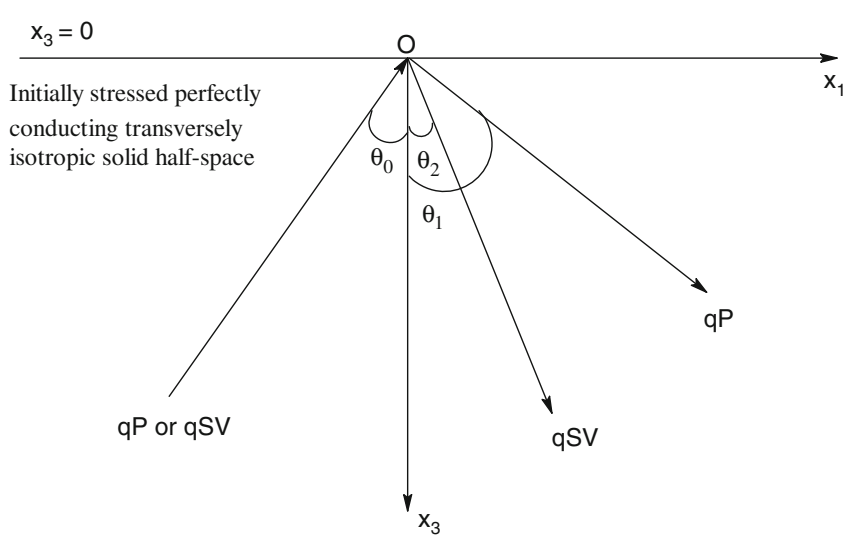

Figure 1. Geometry of the problem.

The required boundary conditions at free surface $x_{3}=0$ are:

$$
\tau_{33}+\bar{\tau}_{33}=0, \tau_{31}+\bar{\tau}_{31}=0, \quad \text { at } x_{3}=0
$$

where $\bar{\tau}_{i j}=\mu_{e}\left[h_{i} h_{j}+h_{j} h_{i}-H_{k} h_{k} \delta_{i j}\right]$.

The displacement potentials of incident and reflected waves given by equations (17) and (18) will satisfy the boundary conditions (equation 21) at $x_{3}=0$, if the following relations hold

$$
\begin{aligned}
& \frac{\sin \theta_{0}}{V_{0}}=\frac{\sin \theta_{1}}{V_{1}}=\frac{\sin \theta_{2}}{V_{2}}, \\
& K_{0} V_{0}=K_{1} V_{1}=K_{2} V_{2}=\omega \text { (say), }
\end{aligned}
$$

and we obtain the following reflection coefficients.

(a) For incident $\mathrm{qP}$ wave

$$
\begin{gathered}
A_{1}=-\frac{\left[\left\{F_{1} F_{2}\left(C-\mu_{e} H_{0}^{2}\right)+\left(F-\mu_{e} H_{0}^{2}\right)\right\}\left\{\sin \theta_{1} \cos \theta_{2}+\cos \theta_{1} \sin \theta_{2}\right\}\right.}{\frac{+}{A_{0}}=-\frac{\left.\left.F_{1}+F_{2}\right)\left\{\left(C-\mu_{e} H_{0}^{2}\right) \cos \theta_{1} \cos \theta_{2}+\left(F-\mu_{e} H_{0}^{2}\right) \sin \theta_{1} \sin \theta_{2}\right\}\right]}{\left[\left\{\left(F-\mu_{e} H_{0}^{2}\right)-F_{1} F_{2}\left(C-\mu_{e} H_{0}^{2}\right)\right\}\left\{\sin \theta_{1} \cos \theta_{2}-\cos \theta_{1} \sin \theta_{2}\right\}\right.},} \\
\left.+\left(F_{1}-F_{2}\right)\left\{\left(C-\mu_{e} H_{0}^{2}\right) \cos \theta_{1} \cos \theta_{2}-\left(F-\mu_{e} H_{0}^{2}\right) \sin \theta_{1} \sin \theta_{2}\right\}\right] \\
\frac{\left[\left\{\left(F-\mu_{e} H_{0}^{2}\right)+F_{1}^{2}\left(C-\mu_{e} H_{0}^{2}\right)\right\}\left\{\sin \theta_{1} \cos \theta_{1}\right\}\right.}{A_{0}}=2 \frac{V_{2}}{V_{1}} \frac{\left.+F_{1}\left\{\left(C-\mu_{e} H_{0}^{2}\right) \cos ^{2} \theta_{1}+\left(F-\mu_{e} H_{0}^{2}\right) \sin ^{2} \theta_{1}\right\}\right]}{\left[\left\{\left(F-\mu_{e} H_{0}^{2}\right)-F_{1} F_{2}\left(C-\mu_{e} H_{0}^{2}\right)\right\}\left\{\sin \theta_{1} \cos \theta_{2}-\cos \theta_{1} \sin \theta_{2}\right\}\right.} \\
\left.+\left(F_{1}-F_{2}\right)\left\{\left(C-\mu_{e} H_{0}^{2}\right) \cos \theta_{1} \cos \theta_{2}-\left(F-\mu_{e} H_{0}^{2}\right) \sin \theta_{1} \sin \theta_{2}\right\}\right]
\end{gathered} .
$$

(b) For incident qSV wave

$$
\begin{aligned}
& {\left[\left\{\left(C-\mu_{e} H_{0}^{2}\right) \cos ^{2} \theta_{2}+\left(F-\mu_{e} H_{0}^{2}\right) \sin ^{2} \theta_{2}\right\} F_{2}\right.} \\
& \frac{A_{1}}{A_{0}}=-2\left(\frac{V_{1}}{V_{2}}\right) \frac{\left.+\left\{F_{2}^{2}\left(C-\mu_{e} H_{0}^{2}\right)+\left(F-\mu_{e} H_{0}^{2}\right)\right\} \sin \theta_{2} \cos \theta_{2}\right]}{\left[\left\{\left(F-\mu_{e} H_{0}^{2}\right)-F_{1} F_{2}\left(C-\mu_{e} H_{0}^{2}\right)\right\}\left\{\sin \theta_{1} \cos \theta_{2}-\cos \theta_{1} \sin \theta_{2}\right\}\right.} \text {, } \\
& \left.+\left(F_{1}-F_{2}\right)\left\{\left(C-\mu_{e} H_{0}^{2}\right) \cos \theta_{1} \cos \theta_{2}-\left(F-\mu_{e} H_{0}^{2}\right) \sin \theta_{1} \sin \theta_{2}\right\}\right] \\
& {\left[\left\{\left(F-\mu_{e} H_{0}^{2}\right)+F_{1} F_{2}\left(C-\mu_{e} H_{0}^{2}\right)\right\}\left\{\sin \theta_{1} \cos \theta_{2}+\sin \theta_{2} \cos \theta_{1}\right\}\right.} \\
& \frac{A_{2}}{A_{0}}=\frac{\left.+\left(F_{1}+F_{2}\right)\left\{\left(C-\mu_{e} H_{0}^{2}\right) \cos \theta_{1} \cos \theta_{2}+\left(F-\mu_{e} H_{0}^{2}\right)\right\} \sin \theta_{1} \sin \theta_{2}\right]}{\left[\left\{\left(F-\mu_{e} H_{0}^{2}\right)-F_{1} F_{2}\left(C-\mu_{e} H_{0}^{2}\right)\right\}\left\{\sin \theta_{1} \cos \theta_{2}-\cos \theta_{1} \sin \theta_{2}\right\}\right.} . \\
& \left.+\left(F_{1}-F_{2}\right)\left\{\left(C-\mu_{e} H_{0}^{2}\right) \cos \theta_{1} \cos \theta_{2}-\left(F-\mu_{e} H_{0}^{2}\right) \sin \theta_{1} \sin \theta_{2}\right\}\right]
\end{aligned}
$$




\section{Energy ratios}

Following Achenbach (1976), we obtain the energy ratios of reflected $\mathrm{qP}$ and $\mathrm{qSV}$ waves as follows:

(a) Incident $\mathrm{qP}$ wave,

$$
\begin{aligned}
E_{1} & =-\left(\frac{A_{1}}{A_{0}}\right)^{2}, \\
E_{2} & =-\left(\frac{A_{2}}{A_{0}}\right)^{2} \frac{V_{1}}{V_{2}} \\
& \times\left[\frac{\left(L+C F_{2}^{2}\right) \cos \theta_{2}+F_{2}(L+F) \sin \theta_{2}}{\left(L+C F_{1}^{2}\right) \cos \theta_{1}+F_{1}(L+F) \sin \theta_{1}}\right],
\end{aligned}
$$

(b) Incident qSV wave,

$$
\begin{aligned}
E_{1} & =-\left(\frac{A_{1}}{A_{0}}\right)^{2} \frac{V_{2}}{V_{1}} \\
& \times\left[\frac{\left(L+C F_{1}^{2}\right) \cos \theta_{1}+F_{1}(L+F) \sin \theta_{1}}{\left(L+C F_{2}^{2}\right) \cos \theta_{2}+F_{2}(L+F) \sin \theta_{2}}\right], \\
E_{2} & =-\left(\frac{A_{2}}{A_{0}}\right)^{2} .
\end{aligned}
$$

\section{Numerical results and discussion}

To compute the absolute values of reflection coefficients and energy ratios of reflected waves, the following relevant parameters of Magnesium material are considered

$$
\begin{aligned}
\rho & =1.74 \mathrm{~kg} \mathrm{~m}^{-3}, & A & =5.974 \times 10^{10} \mathrm{~N} \mathrm{~m}^{-2}, \\
F & =2.17 \times 10^{10} \mathrm{~N} \mathrm{~m}^{-2}, & C & =6.17 \times 10^{10} \mathrm{~N} \mathrm{~m}^{-2}, \\
L & =1.639 \times 10^{10} \mathrm{~N} \mathrm{~m}^{-2} . & &
\end{aligned}
$$

With the help of a FORTRAN program, the reflection coefficients and energy ratios of various quasi plane waves are computed against the angle of incidence of $\mathrm{qP}$ or qSV wave. These variations of the reflection coefficients and energy ratios of reflected waves are shown graphically in figures $2-8$.

The variations of the reflection coefficients of reflected $\mathrm{qP}$ and $\mathrm{qSV}$ waves are shown graphically in figure 2 against the angle of incidence of $\mathrm{qP}$ wave for $p_{0}=0, p_{0}=0.5 \times 10^{10} \mathrm{~N} \mathrm{~m}^{-2}$ and $p_{0}$ $=1.0 \times 10^{10} \mathrm{~N} \mathrm{~m}^{-2}$, when $H_{0}=0.1 \times 10^{5} \mathrm{Oe}$. For $p_{0}=0$, the reflection coefficient of $\mathrm{qP}$ wave is one for normal incidence and it decreases sharply to value 0.1458 at $\theta_{0}=63^{\circ}$. Thereafter, it increases sharply to its maximum value one at grazing incidence. The increase in value of initial stress parameter increases the reflection coefficient of the reflected $\mathrm{qP}$ wave at all angles of incidence except the normal and grazing incidence. The maximum effect of initial stress on reflected $\mathrm{qP}$ wave is observed at angles near $\theta_{0}=63^{\circ}$. For $p_{0}=0$, the reflection coefficient of qSV wave is 2.26 for normal incidence and it decreases sharply to its minimum value zero at grazing incidence. The increase in the value of initial stress parameter decreases the reflection coefficient of qSV wave at all angles of incidence except the grazing incidence. The maximum effect of initial stress is observed at angles near normal incidence.

The variations of the reflection coefficients of reflected $\mathrm{qP}$ and $\mathrm{qSV}$ waves are shown graphically in figure 3 against the angle of incidence of $\mathrm{qP}$ wave for $H_{0}=0, H_{0}=0.5 \times 10^{5}$ Oe and $H_{0}=1.0 \times$ $10^{5} \mathrm{Oe}$, when $p_{0}=0.5 \times 10^{10} \mathrm{~N} \mathrm{~m}^{-2}$. For $H_{0}=0$, the reflection coefficient of $\mathrm{qP}$ wave is one for normal incidence and it decreases sharply to value 0.2676 at $\theta_{0}=61^{\circ}$. Thereafter, it increases sharply to its maximum value one at grazing incidence. The increase in value of magnetic field parameter decreases the reflection coefficient of the reflected $\mathrm{qP}$ wave at all angles of incidence except the normal and grazing incidence. The maximum effect of initial stress on reflected $\mathrm{qP}$ wave is observed at angles near $\theta_{0}=61^{\circ}$. For $H_{0}=0$, the reflection coefficient of qSV wave is 1.96 for normal incidence and it decreases sharply to its minimum value zero at grazing incidence. The increase in the value of magnetic field parameter decreases the reflection coefficient of qSV wave at all angles of incidence except the grazing incidence. The maximum effect of initial stress is observed at angles near normal incidence.

For incidence of $\mathrm{qP}$ wave, the energy ratios of reflected $\mathrm{qP}$ and $\mathrm{qSV}$ waves are also computed and shown graphically in figures 4 and 5. The comparison of solid and dashed lines in these figures shows the effects of initial stress and magnetic field parameters on the energy ratios of reflected $\mathrm{qP}$ and qSV waves.

The variations of the reflection coefficients of reflected qP and qSV waves are shown graphically in figure 6 against the angle of incidence of qSV wave for $p_{0}=0, p_{0}=0.5 \times 10^{10} \mathrm{~N} \mathrm{~m}^{-2}$ and $p_{0}=$ $1.0 \times 10^{10} \mathrm{~N} \mathrm{~m}^{-2}$, when $H_{0}=0.1 \times 10^{5}$ Oe. For $p_{0}=0$, the reflection coefficient of $\mathrm{qP}$ wave is zero for normal incidence and it increases to its maximum value one at the critical angle $\theta_{0}=34^{\circ}$. The increase in initial stress parameter results in early critical angles of incidence. The effect of initial stress is zero at normal incidence and it increases with the increase in the angle of incidence.

For $p_{0}=0$, the reflection coefficient of qSV wave is one for normal incidence and it decreases with the increase in angle of incidence. The comparison of solid and dashed lines in figure 6 shows the 

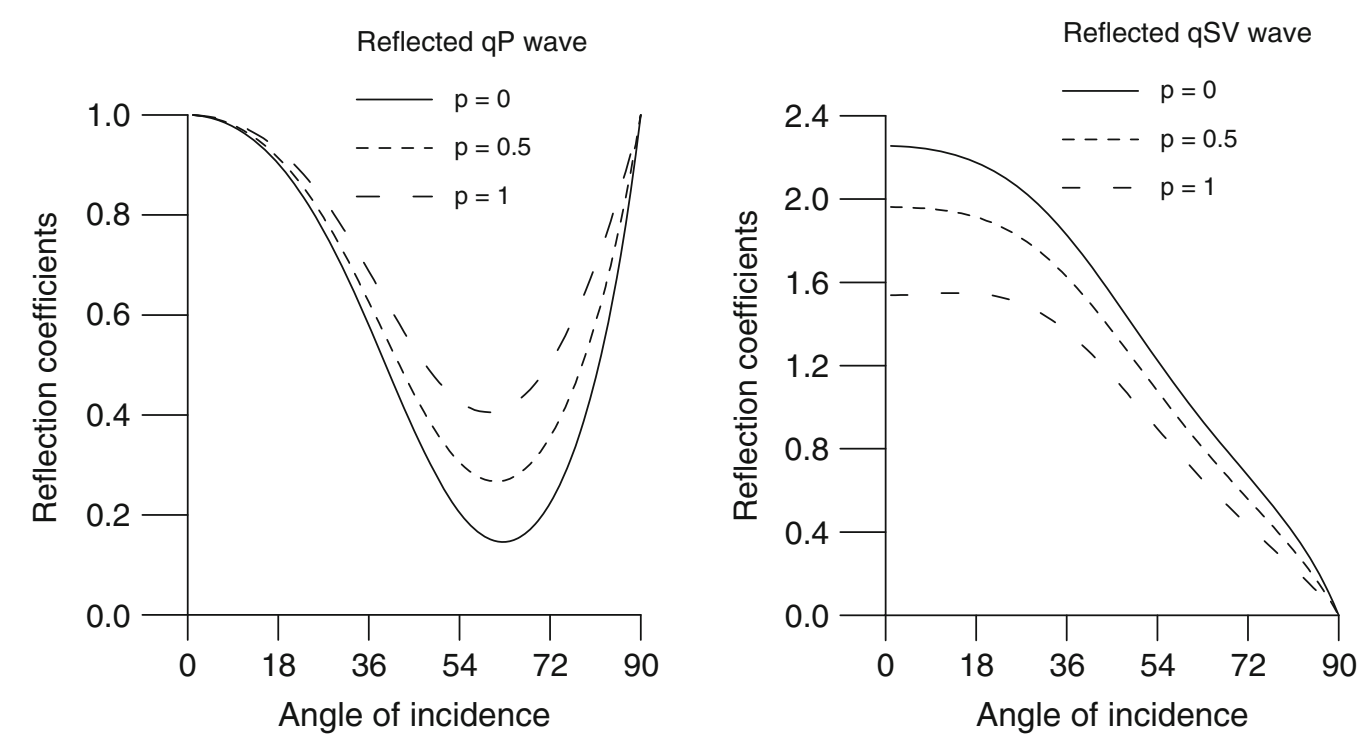

Figure 2. Effect of initial stress parameter on the reflection coefficients for incident qP wave, when $H_{0}=0.1 \times 10^{5}$ Oe.
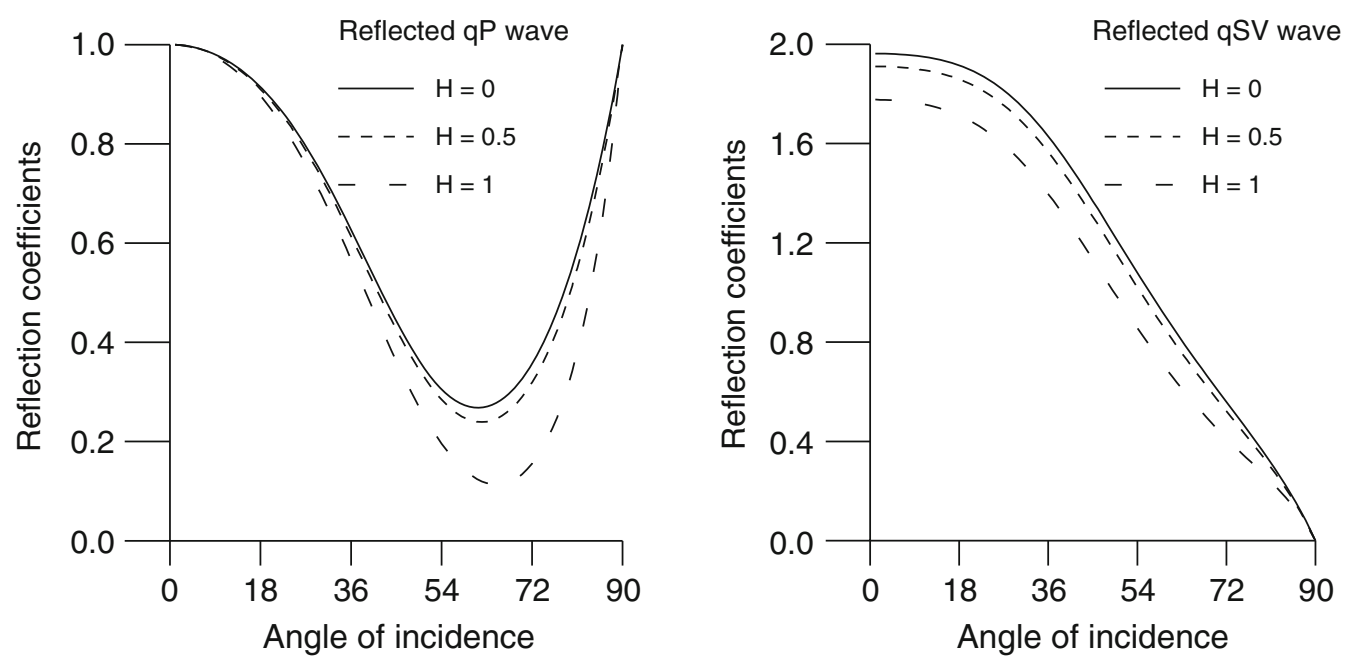

Figure 3. Effect of magnetic field on the reflection coefficients for incident $\mathrm{qP}$ wave, when $p_{0}=0.5 \times 10^{10} \mathrm{~N} \mathrm{~m}^{-2}$.
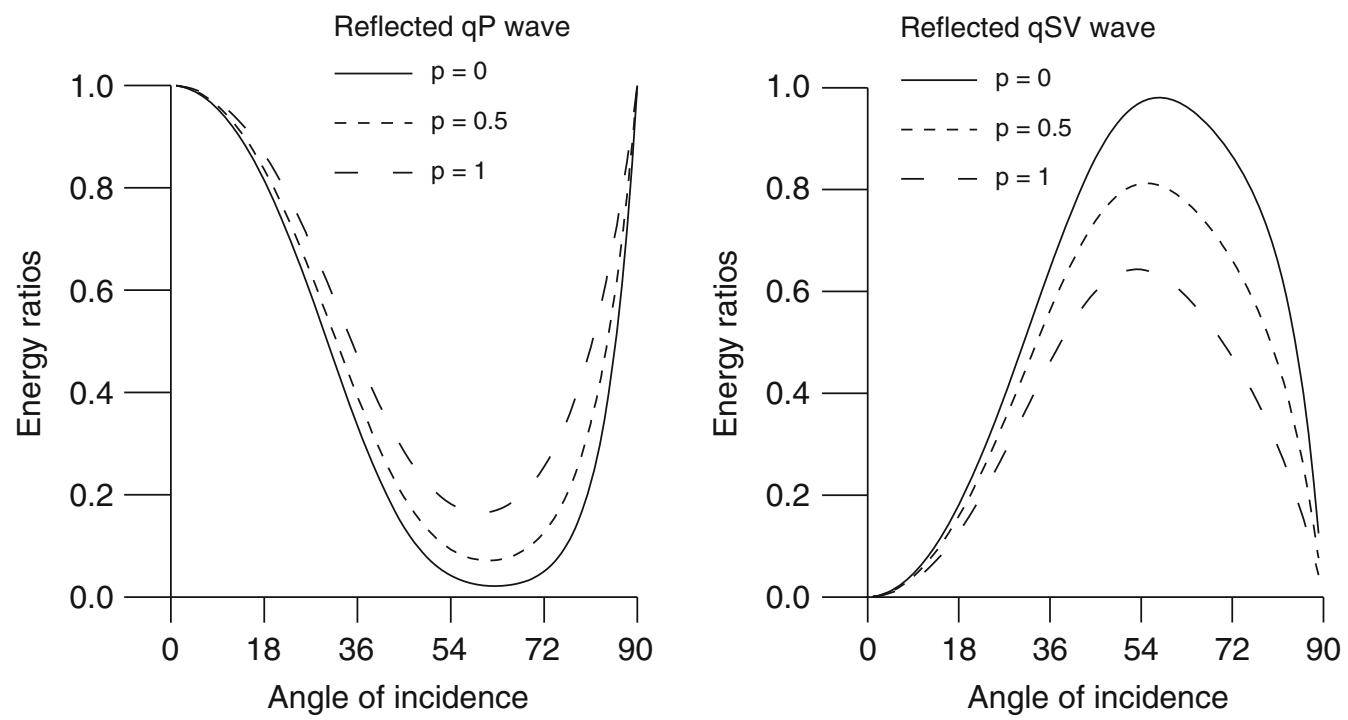

Figure 4. Effect of initial stress parameter on the energy ratios of reflected waves for incident qP wave, when $H_{0}=0.1 \times 10^{5}$ Oe. 

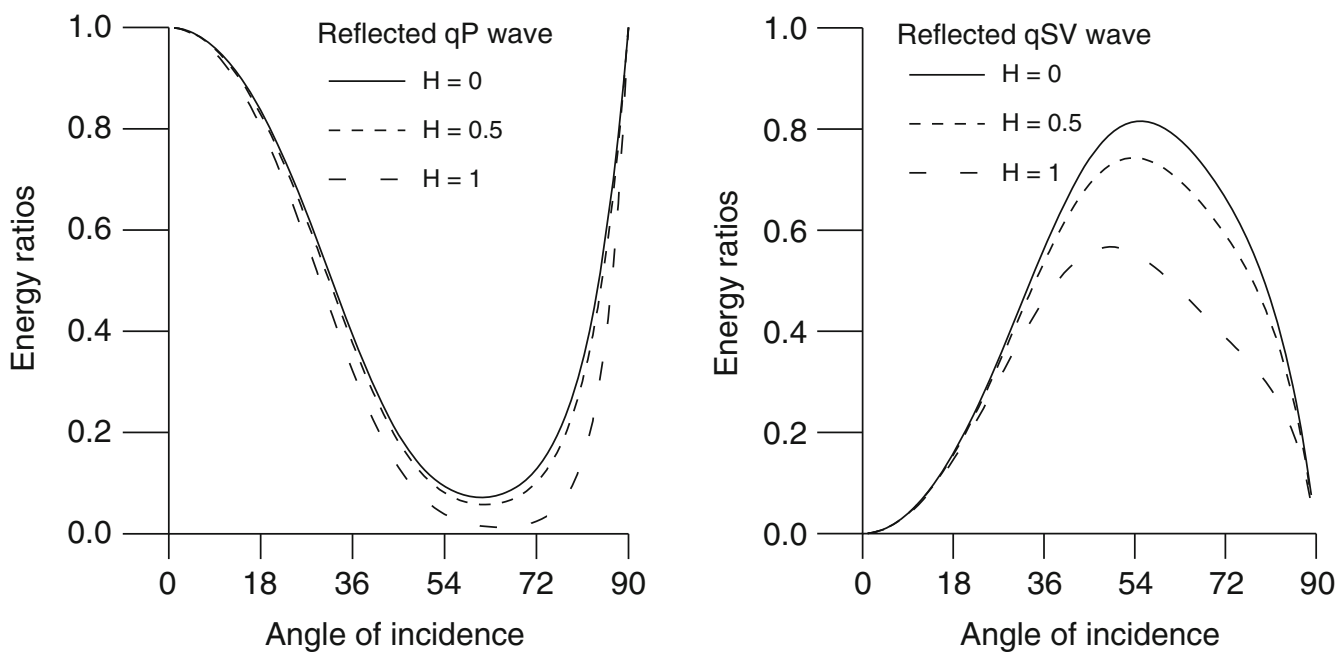

Figure 5. Effect of magnetic field on the energy ratios of reflected waves for incident qP wave, when $p_{0}=0.5 \times 10^{10} \mathrm{~N} \mathrm{~m}^{-2}$.
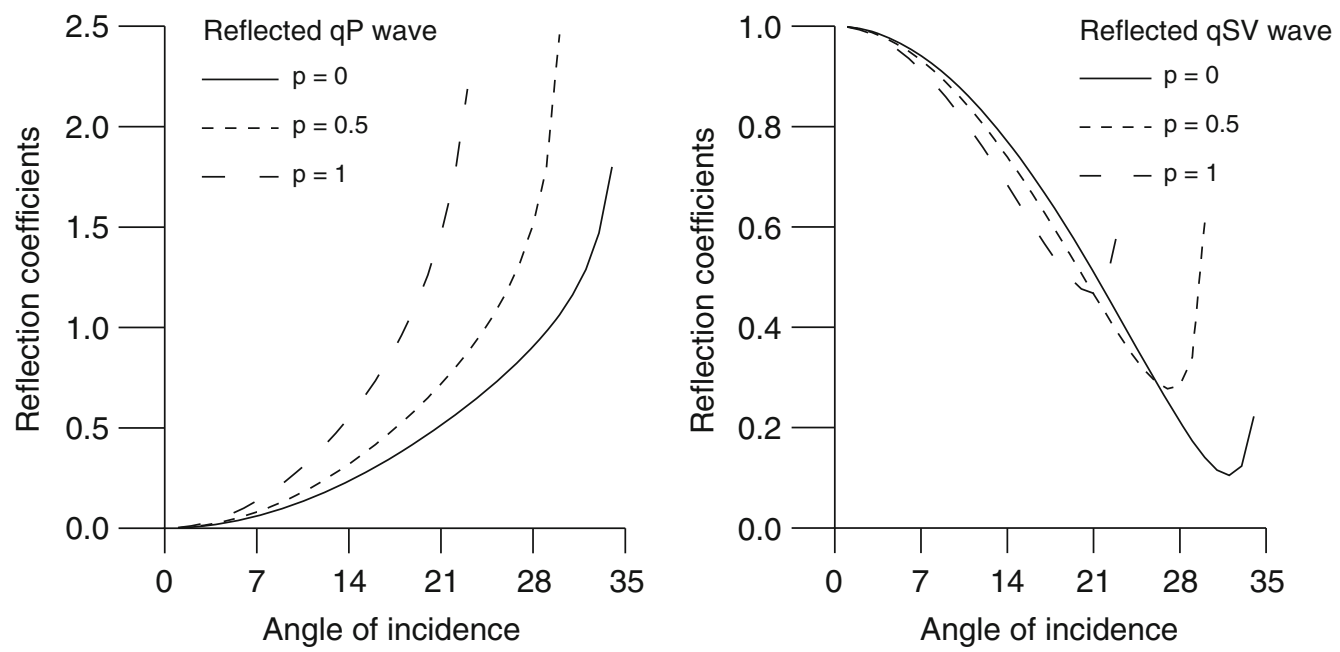

Figure 6. Effect of initial stress parameter on the reflection coefficients for incident qSV wave, when $H_{0}=0.1 \times 10^{5}$ Oe.
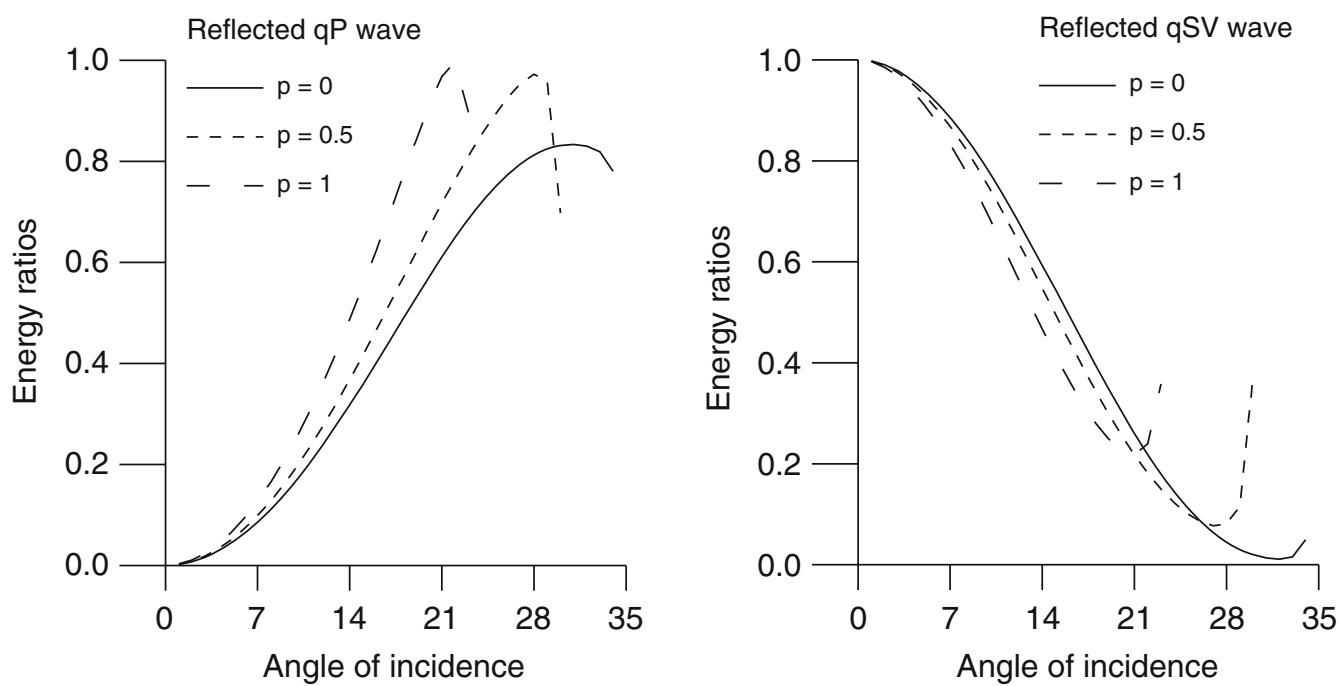

Figure 7. Effect of initial stress parameter on the energy ratios of reflected waves for incident qSV wave, when $H_{0}=0.1 \times 10^{5}$ Oe. 

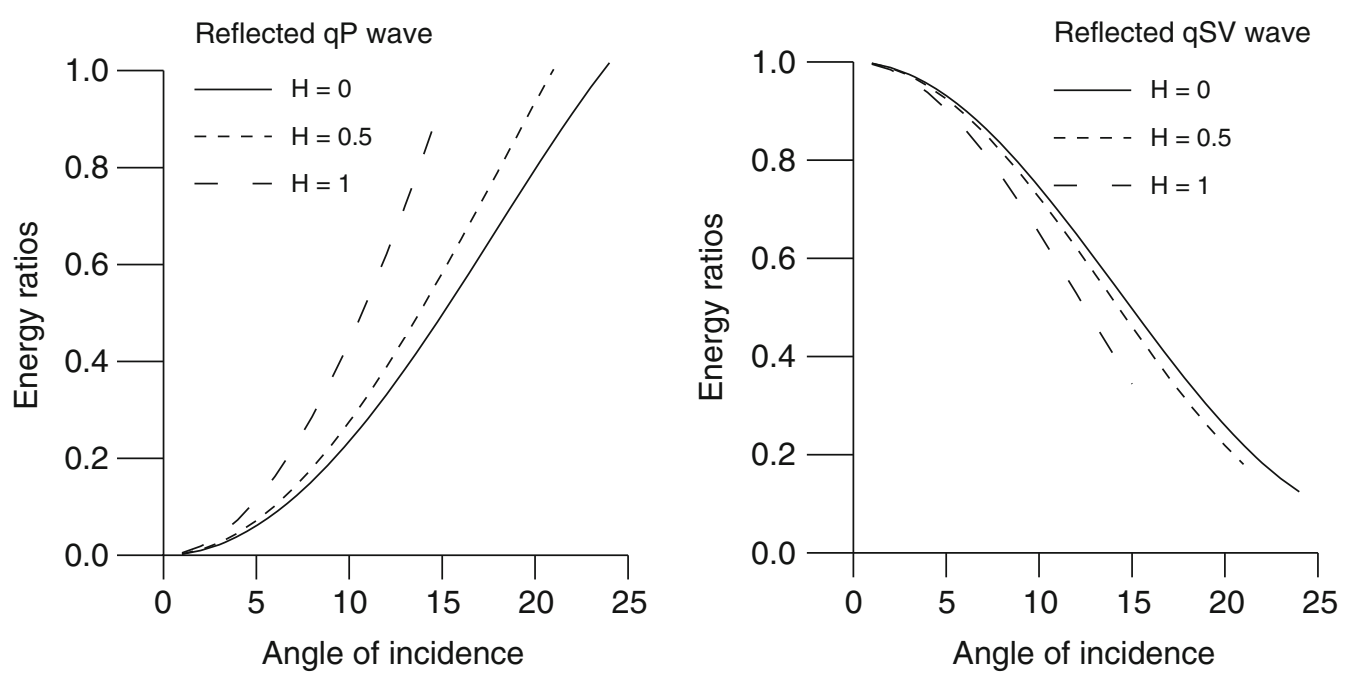

Figure 8. Effect of magnetic field parameter on the energy ratios of reflected waves for incident qSV wave, when $p_{0}=0.5 \times$ $10^{10} \mathrm{~N} \mathrm{~m}^{-2}$.

effect of initial stress on the reflection coefficient of reflected qSV wave.

For incidence of $\mathrm{qP}$ wave, the energy ratios of reflected $\mathrm{qP}$ and $\mathrm{qSV}$ waves are also computed and shown graphically in figures 7 and 8 . The comparison of solid and dashed lines in these figures shows the effect of initial stress and magnetic field parameters on the energy ratios of reflected $\mathrm{qP}$ and $\mathrm{qSV}$ waves.

The numerical example discussed above implies that the mathematical model solved in this work may be used to compute the exact effects of initial stress and magnetic field on propagation characteristics of the waves at the boundary of the transversely isotropic solid half-space. The anisotropy in the model should be viewed as means of investigating the internal constitution of the rocks, materials, structures, etc. Initial stress in the model represents in situ stress distribution. The numerical results discussed in the paper are based on a particular numerical model and may not qualify for quantitative generalisation. However, the qualitative interpretation of these results may help to extract the following few useful conclusions: The effects of initial stress and magnetic field on the reflected waves vary with the direction of the incident wave, elastic properties and the anisotropy present in the medium. Energy partition among reflected waves is more sensitive to the presence of initial stress and magnetic field as compared to other propagation characteristics. The work presented relates the geophysical properties (anisotropy, initial-stress) of a realistic medium to its propagation characteristics (reflection coefficients and energy ratios). When supported with a real data, such a relation may be used to understand a variety of geophysical problems.

\section{Conclusion}

The governing equations of a perfectly conducting transversely isotropic elastic solid with initial stress are solved to obtain the velocity equation which indicates the existence of two quasi plane waves in the medium. Reflection coefficients and energy ratios for reflected $\mathrm{qP}$ and $\mathrm{qSV}$ waves are computed numerically for Magnesium material. The initial stress and magnetic field parameters affect significantly the reflection coefficients and energy ratios.

\section{References}

Acharya H K 1970 Reflection from the free surface of inhomogeneous media; Bull. Seismol. Soc. Am. 60 1101-1104.

Achenbach J D 1976 Wave propagation in elastic solids; North Holland Publishing Company, New York.

Ahmad F and Khan A 2001 Effect of rotation on wave propagation in a transversely isotropic medium; Math. Probab. Eng. 7 147-154.

Auld B A 1973 Acoustic Field and waves in solids; Wiley, New York.

Biot M A 1965 Mechanics of Incremental Deformations; Wiley, New York.

Chattopadhyay A and Rogerson G A 2001 Wave reflection in slightly compressible, finitely deformed elastic media; Archives of Applied Mechanics 71 307-316.

Das S C, Sengupta S and Acharya 1994 D P Magnetovisco-elastic waves in an initially stressed conducting layer including strain rate and stress rate; Bull. Tech. Univ. Island $47243-255$.

De S N and Sengupta P R 1972 Magneto elastic waves and disturbances in initially stressed conducting media; Pure Appl. Geophys. 93 41-54.

Dey S and Addy S K 1979 Reflection and refractional plane waves under initial stresses at an interface; Int. J. NonLinear. Mech. 14(2) 101-110. 
Gutenberg B 1944 Energy ratio of reflected and refracted seismic waves; Bull. Seismol. Soc. Am. 34 85-112.

Keith C M and Crampin S 1977 Seismic body waves in anisotropic media, reflection and refraction at a plane interface; Geophys. J. Roy. Astron. Soc. 49 181-208.

Knott C G 1899 Reflection and refraction of elastic waves with seismological applications; Phil. Mag. 48 64-97.

Love A E H 1942 A treaties on the mathematical theory of elasticity; Cambridge Univ. Press, London.

Norris A N 1983 Propagation of planes in a pre-stressed elastic medium; J. Acoust. Soc. Am. 74 1642-1643.

Pal A K and Chattopadhyay A 1984 The reflection phenomena of plane waves at a free boundary in a pre-stressed elastic medium; J. Acoust. Soc. Am. 76 924-925.

Rakshit A K and Sengupta P R 1998 Magneto-thermoelastic waves in initially stressed conducting layer; Sadhana 23 233-246.
Selim M M and Ahmed M K 2006 Propagation and attenuation of seismic body waves in dissipative medium under initial and couple stresses; Appl. Math. 182 1064-1074.

Sharma M D 2007 Effect of initial stress on reflection at the free surfaces of anisotropic elastic medium; J. Earth Syst. Sci. 116(6) 537-551.

Singh B 2010 Wave propagation in a prestressed piezoelectric half-space; Acta Mech. 211 337-344.

Tolstoy I 1982 On elastic waves in pre-stressed solids; J. Geophys. Res. 87 6823-6827.

Tooly R D, Spancer T W and Sagoci H F 1965 Reflection and transmission of plane compression waves; Geophysics $30552-570$.

Yu C P and Tang S 1966 Magneto-elastic waves in initially stressed conductors; Z. Angew. Math. Phys. 17 $766-775$.

MS received 22 December 2011; revised 19 November 2012; accepted 1 February 2013 